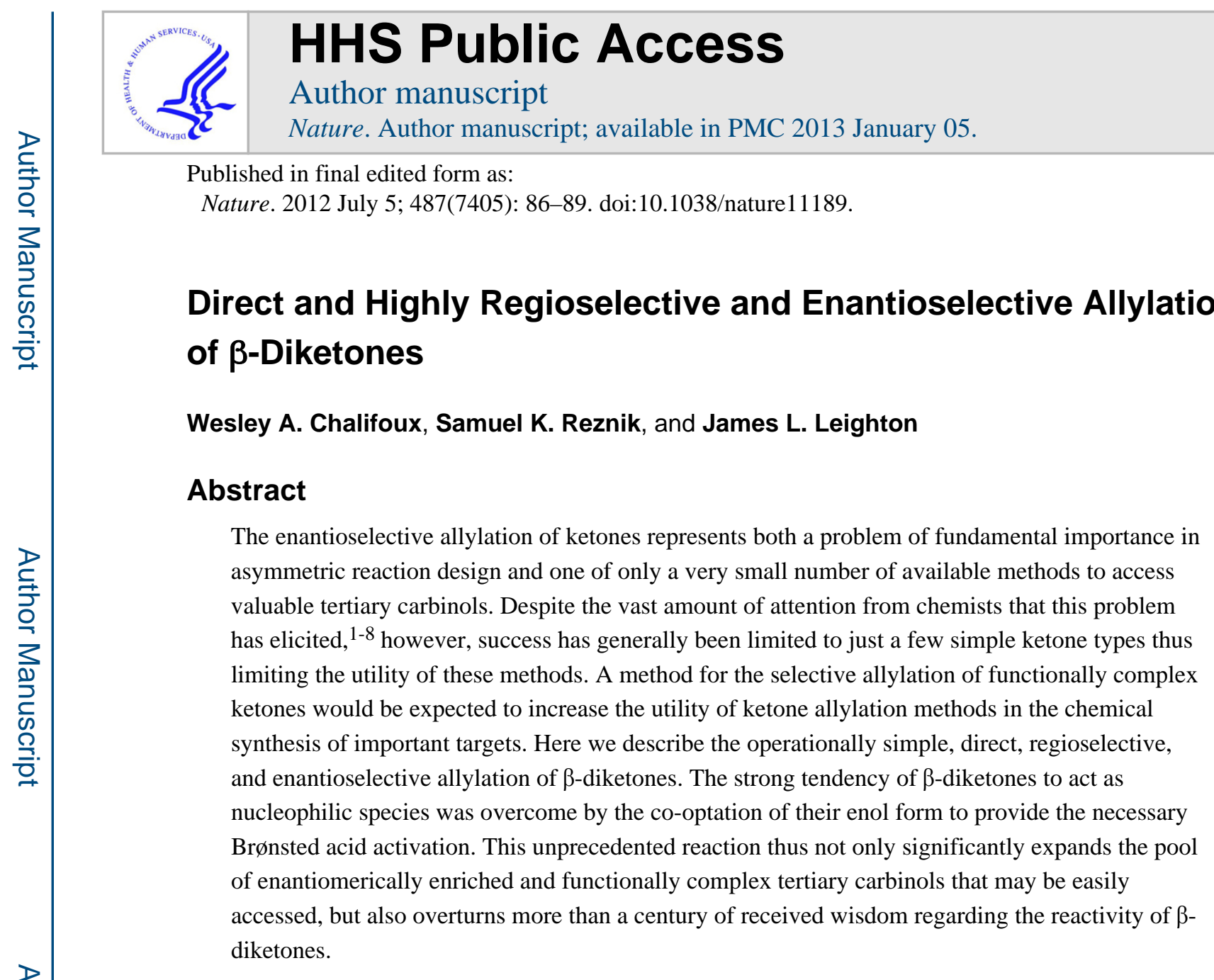

Chiral non-racemic tertiary carbinols appear with some frequency in important natural products and in medicinal chemistry programs. Asymmetric ketone allylation represents one of the few methods available for their direct synthesis, and the past 15 years have seen the appearance of several seminal reports that describe some important and significant progress towards the development of efficient, practical, and highly enantioselective methods. ${ }^{1-8}$ With only a few exceptions, however, these methods are limited in scope to aryl methyl ketones and aryl linear alkyl ketones (i.e. $\mathrm{ArCOCH}_{2} \mathrm{R}$ ). Thus, while efficiency and practicality rightly constitute one major focus in asymmetric reaction design, scope, generality, reliability, and applicability in complex settings are equally vital to the realization of methods with truly broad utility and that enjoy widespread adoption. ${ }^{9}$

Users may view, print, copy, download and text and data- mine the content in such documents, for the purposes of academic research, subject always to the full Conditions of use: http://www.nature.com/authors/editorial_policies/license.html\#terms

Supplementary Information is linked to the online version of the paper at www.nature.com/nature.

Author contributions W.A.C. planned and carried out the vast majority of the experimental work. S.K.R. carried out the initial experiments that established the validity of the idea and optimized the allylation of acetylacetone. J.L.L. conceived and directed the project and wrote the manuscript.

Author Information X-ray crystallographic data have been deposited in the Cambridge Crystallographic Data Centre database (http:// www.ccdc.cam.ac.uk/) under accession code CCDC 874744. Reprints and Permissions information is available at www.nature.com/ reprints. The authors declare no competing financial interests. Readers are welcome to comment on the online version of this article at www.nature.com/nature. Correspondence and requests for materials should be made to J.L.L. (leighton@chem.columbia.edu). 
These thoughts were triggered by our retrosynthesis of the $\mathrm{AB}$ spiroketal portion of the extraordinarily potent and precious anti-mitotic marine macrolide spongistatin $1^{10-12}$ which bears a tertiary carbinol - to $\beta$-hydroxyketone 1 (Fig. 1a). It seemed a tantalizingly straightforward proposition that the ideal ${ }^{13}$ synthesis of $\mathbf{1}$ would involve a direct asymmetric allylation of acetylacetone (Fig. 1b). While a few sporadic examples of the non-asymmetric allylation of acetylactone have been reported, ${ }^{14-16}$ however, there are no known examples of an enantioselective addition of a nucleophile to a $\beta$-diketone - indeed, there are simply no general methods that employ $\beta$-diketones as electrophiles in carbon-carbon bond forming reactions at all. This is unsurprising as $\beta$-diketones are not simply compounds with two ketones, but rather are distinct chemical entities that exist primarily in their enol tautomer form, and to the extent that they are employed at all in organic chemical synthesis it is typically as nucleophiles in, for example, simple alkylation reactions or Knoevenagel condensations. As a result, an asymmetric allylation approach to the synthesis of $\mathbf{1}$ using known methods would require protection/masking of one of the ketones thus turning an ideal synthesis in principle into a multi-step process involving protecting group manipulations and/or oxidation state adjustments in practice. While the development of a method for the direct asymmetric allylation of acetylacetone seemed a worthy enough goal, it was of course the generalization of such a method to include a broad range of $\beta$-diketones, including unsymmetrical $\beta$-diketones, that became the ultimate goal of this study. Far from representing a straightforward extension of the proposed method, however, unsymmetrical $\beta$-diketones pose an additional distinct and formidable challenge in reaction design, and that is the control of regioselectivity (Fig. 1c). Intrigued by these challenges and by the almost total dearth of useful precedent, we have pursued a general, practical, and highly regioselective and enantioselective allylation of $\beta$-diketones and report herein its successful development and mechanistic elucidation.

Rather than attempting to mask in situ, preclude the formation of, or otherwise render innocuous the enol tautomer of the $\beta$-diketone, our approach centered on the idea that we might instead use it to generate, by reaction with an allylchlorosilane, $\beta$-silyloxyenone $\mathbf{2}$ which would subsequently undergo intramolecular allylation (Fig. 2a). This otherwise beneficial tethering strategy would in this case also lead to deactivation of the ketone electrophile by converting it into a vinylogous silyl ester, however, raising concerns about whether the desired reaction might be impractically slow and allow side reactions (e.g. Knoevenagel condensation between $\mathbf{2}$ and unreacted diketone, or intermolecular diketone allylation by the allylsilane or by 2 ) to compete. Ideally then, to favor the desired pathway, the complexation should be very fast so that no unreacted diketone is available for such side reactions, and the allylsilane should become strongly activated upon complexation.

Allylsilane 3 and other related aminosilanes are strongly activated by protonation with Brønsted acids, ${ }^{17,18}$ and given that the proposed enol silylation reaction would produce an equivalent of $\mathrm{HCl}$, it seemed possible that $\mathbf{3}$ - which is wholly incapable of allylating ketones - might be perfectly configured to react with $\beta$-diketones by way of activated silane complex 4 (Fig. 2a). Indeed, we were delighted to find that allylsilane 3 smoothly allylates acetylacetone, and, upon optimization of the reaction conditions $\left(\mathrm{CHCl}_{3}, 23{ }^{\circ} \mathrm{C}, 1 \mathrm{~h}\right)$, produced 1 in $72 \%$ yield and $89 \%$ ee (Fig. 2b). This operationally simple reaction both provides direct and scalable access to $\mathbf{1}$ from two commercially available compounds, and, 
along with the observation that neither $\mathbf{5}$ nor $\mathbf{6}$ were allylated by $\mathbf{3}$ (Supplementary Fig. 1 and 2), strongly implies that the underlying mechanistic proposal is sound. The enol form of the diketone, which would typically represent a serious functional group incompatibility, has thus been co-opted as the very functionality responsible for the success of the reaction.

With this encouraging proof of concept in hand, we initiated an examination of the scope of the reaction (Fig. 3a). We began with three additional symmetrical $\beta$-diketones, and as shown, products $\mathbf{7 , 8}$, and $\mathbf{9}$ were all obtained in good yields and with good to excellent levels of enantioselectivity, establishing that both $t$-butyl and aryl ketones are well tolerated (Fig. 3b). A series of unsymmetrical $\beta$-diketones were examined next in an effort to establish whether and under what circumstances useful levels of regioselectivity could be realized. While substrates that pitted two alkyl ketones against each other led to little or no regioselectivity, it was quickly discovered that substrates that pitted a conjugated ketone against an alkyl ketone consistently reacted such that the allyl group was added to the alkyl ketone with excellent regio- and enantioselectivity (Fig. 3c). This preference not only led to the efficient and selective production of 10-12, but also was operative even when the alkyl group is a $t$-butyl group ( $c f$. 13). Differentially substituted 1,3-diphenylpropane-1,3-diones were examined next and we were initially disappointed to find that the para-bromo, paramethoxy derivative was completely non-selective. When the impact of ortho substitution was interrogated, however, quite surprising and subtle effects were revealed (Fig. 3d). Thus, whereas an ortho-bromo group regioselectively directs the allylation to the distal ketone ( $c f$. 14 and 15), an ortho-methoxy group has the opposite effect ( $c f .16$ ) albeit with only moderate (4:1) regioselectivity. These effects are mutually reinforcing, as demonstrated by the reaction of the ortho-bromo, ortho-methoxy analog to give $\mathbf{1 7}$ as a single regioisomer in $89 \%$ yield and $95 \%$ ee, a result that is particularly striking in light of the complete lack of regioselectivity in the reaction of the corresponding para, para isomer. Finally, two additional examples ( $c f . \mathbf{1 8}$ and 19) illustrate that this ortho-effect extends to groups other than bromide, and establishes that it is the methoxy group that is the outlier (cf. 16).

In principle, the extension of this reaction methodology to crotylation reactions would allow the establishment of a second stereocenter in the allylic position of the homoallylic alcohol products, but examples of highly diastereo- and enantioselective ketone crotylation reactions are rare. ${ }^{5,7,8,19}$ We therefore decided to interrogate the ability of crotylsilanes $\mathbf{2 0}$ and $\mathbf{2 1}^{20}$ to crotylate $\beta$-diketones, and it was found that treatment of acetylacetone and benzoylacetone with trans-crotylsilane $\mathbf{2 0}$ led to the isolation of $\mathbf{2 2}(69 \%, 89 \%$ ee) and $\mathbf{2 3}$ (75\%, 97\% ee), respectively (Fig. 4a). Unexpectedly, however, treatment of benzoylacetone with ciscrotylsilane 21 resulted in a much slower reaction that provided the desired product in very low yield $(<15 \%)$ along with significant amounts of a byproduct we have tentatively identified as $\mathbf{2 4}$. The formation of $\mathbf{2 4}$ is readily rationalized as the result of a Knoevenagel condensation cascade that presumably begins with attack of the silyl enol ether complex on unreacted $\beta$-diketone. This hypothesis implied that the complexation event is slow in these reactions in general and that the desired $\mathrm{C}-\mathrm{C}$ bond-forming step is especially slow in this particular reaction. In this way, the silyl enol ether complex and the starting diketone would both be present in significant concentrations for long periods of time. One solution suggested by this analysis was to identify a way to accelerate the complexation so that no 
unreacted diketone would be available to the silyl enol ether complex, and it seemed plausible that replacement of the chloride on the silane with a triflate $\left({ }^{-} \mathrm{OSO}_{2} \mathrm{CF}_{3}\right)$ might accomplish this. Indeed, pre-treatment of 21 with $\mathrm{AgOSO}_{2} \mathrm{CF}_{3}$ prior to the addition of acetylacetone and benzoylacetone led to vastly improved results, giving $\mathbf{2 5}$ and $\mathbf{2 6}$, respectively, in excellent yields and enantioselectivities.

With effective conditions worked out for the crotylation reactions, we turned next to an examination of 2-acetyltetralone $\mathbf{2 7}$ as a probe of the impact of substitution on the a-carbon (Fig. 4b). It will be noted that the initially formed products in these reactions are silyl enol ethers (e.g. 28), which are converted to enols that subsequently tautomerize to ketones upon quench and workup. When the a-carbon is substituted, as in initial products $\mathbf{2 8}$, the tautomerization has stereochemical consequences, and we have demonstrated in similar systems that good to excellent diastereocontrol may be realized with proper choice of quench/workup conditions. ${ }^{21}$ The standard quench for the present method entails cooling the reaction mixtures to $-40{ }^{\circ} \mathrm{C}$, and adding $n$-Bu $\mathrm{Bu}_{4} \mathrm{NF}$, followed by an aqueous workup. When the crotylation reactions of $\mathbf{2 7}$ with $\mathbf{2 0}$ and $\mathbf{2 1}$ were quenched and worked up using these conditions, good to excellent diastereoselectivity was realized, resulting in the isolation of 29 and 30 in 69\% and 59\% yield, respectively, with excellent regio- and enantioselectivity. The result is an operationally simple single step and protecting group-free synthesis of complex polyketide-like arrays of three stereocenters (including a tertiary carbinol) from simple and readily available starting materials with exceedingly high levels of enantioselectivity.

We turned next to the elucidation of the mechanistic basis for the remarkable regioselectivity of the reaction. In the reaction of an unsymmetrical $\beta$-diketone with $(S, S)-3$ four different $\beta$-silyloxyenones may be produced $(31 E, 31 Z, 32 E$, and 32Z), which may be considered as two regioisomeric pairs of $E$ and $Z$ isomers (Fig. 5a). After establishing that interconversion between the $E$ and $Z$ isomers in each pair of regioisomers is fast (Supplementary Fig. 3), there are two readily apparent possibilities: 1) there is no (fast) interconversion between $\mathbf{3 1 Z}$ and $\mathbf{3 2 Z}$ and the regioselectivity is determined by the relative rates of formation of $\mathbf{3 1}$ and $\mathbf{3 2}$, and 2) interconversion of $\mathbf{3 1 Z}$ and $\mathbf{3 2 Z}$, presumably by way of $\mathbf{3 3}$, is fast and the regioselectivity is determined by the relative energies of transition states 34 and 35. In order to distinguish between these possibilities, we repeated the reaction of 27 with 21 ( $c f$. Fig. 4b) in $\mathrm{CDCl}_{3}$, and monitored it by ${ }^{1} \mathrm{H}$ NMR spectroscopy (Supplementary Fig. 4). After one hour, the singlet for the methyl group in $\mathbf{2 7}$ disappeared and three new singlets appeared at 2.53, 2.11, and $1.97 \mathrm{ppm}$ in a $~ 2: 3.4: 1$ ratio, and we have assigned these peaks as corresponding to the three possible silyl enol ether complexes. Given that these peaks disappear at the same rate and that this reaction is completely regioselective despite the fact that only one of these silyl enol ethers can react directly to give the product, we conclude that in these reactions the interconversion between $\mathbf{3 1 Z}$ and $32 Z$ is fast ${ }^{22-25}$ and that the regioselectivity is determined by the relative energies of transition states $\mathbf{3 4}$ and $\mathbf{3 5}$, i.e. the reaction is governed by Curtin-Hammett kinetics. ${ }^{26}$

The regioselectivity observed with substrates wherein $\mathrm{R}^{1}=$ aryl/alkenyl and $\mathrm{R}^{2}=$ alkyl $(c f$. Fig. 3c) is difficult to attribute to a simple steric effect given the regioselective production of 
13. Rather, we propose that the energy of transition state $\mathbf{3 4 a}$ is lower than that for $\mathbf{3 5 a}$ because conjugation is maintained in the former pathway but lost completely in the latter (Fig. 5b, Case 1). Conversely, we propose that the regioselectivity observed with substrates wherein both $\mathrm{R}^{1}$ and $\mathrm{R}^{2}$ are aryl groups with at least one of them bearing ortho-substitution (cf. Fig. 3d) is the result of steric effects (Fig. 5b, Case 2). 2-Bromophenyl, mesityl, and 2chloro-3-pyridyl groups are all expected to be substantially rotated out of conjugation with the ketones to which they are attached ${ }^{27-29}$ rendering them highly sterically hindered toward nucleophilic attack, as illustrated in transition state 35b. By contrast, 2-methoxybenzoyl and ortho-unsubstituted phenyl groups are conjugated, ${ }^{30}$ and this allows an unhindered approach

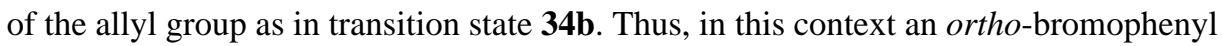
group is effectively large and an ortho-methoxyphenyl group is effectively small, and we believe this steric effect is large enough to overwhelm the complete loss of conjugation in transition state 34b. The moderate regioselectivity for product $\mathbf{1 6}$ is more difficult to rationalize, but may constitute a case where the activation energies for the competing C-C bond forming steps are nearly equivalent and the selectivity derives from a modest shifting of $\mathrm{K}_{\mathrm{eq}}$ towards $31 \mathrm{Z}$ due to the silane preferring to reside away from the larger orthomethoxyphenyl group.

We have demonstrated that it is possible to achieve not only the highly enantioselective allylation and crotylation of $\beta$-diketones, but also the highly regioselective allylation and crotylation of unsymmetrical $\beta$-diketones. The method allows the protecting group-free single step (i.e. ideal) synthesis of functionally and stereochemically complex products from readily available - and undifferentiated - $\beta$-diketone starting materials. Important aspects of the mechanism were elucidated and the regioselectivity was found to be governed by CurtinHammett kinetics. This may have important implications for the extension of this methodology to other dicarbonyl substrate types and experiments along these lines are in progress.

\section{METHODS SUMMARY}

The general procedure for the allylation reactions described in Figure 3 is as follows. To a $0.10 \mathrm{M}$ solution of $\beta$-diketone (1.0 equiv) in anhydrous $\mathrm{CHCl}_{3}$ is added $(S, S)-\mathbf{3}$ (1.2 equiv). The resulting mixture is stirred at ambient temperature $\left(23{ }^{\circ} \mathrm{C}\right)$ for $12-27 \mathrm{~h}$. The mixture is cooled to $-40{ }^{\circ} \mathrm{C}, n$-Bu ${ }_{4} \mathrm{NF}$ (4.0 equiv, $1 \mathrm{M}$ in tetrahydrofuran) is added, and the resulting mixture is maintained at $-40{ }^{\circ} \mathrm{C}$ for $1 \mathrm{~h}$. Saturated aqueous $\mathrm{NH}_{4} \mathrm{Cl}$ is added and the mixture is allowed to warm to ambient temperature. The mixture is extracted 3 times with $\mathrm{CH}_{2} \mathrm{Cl}_{2}$. The combined organic layers are washed with water and brine, dried over $\mathrm{MgSO}_{4}$, filtered, and concentrated. The residue is purified by flash chromatography on silica gel. For complete experimental details and compound characterization, see Supplementary Information.

\section{Supplementary Material}

Refer to Web version on PubMed Central for supplementary material. 


\section{Acknowledgements}

This work was supported by a grant from the National Institute of General Medical Sciences (GM58133). W.A.C. was supported by a National Sciences and Engineering Research Council of Canada (NSERC) Postdoctoral Fellowship. We thank the National Science Foundation (CRIF-0840451) for acquisition of a $400 \mathrm{MHz}$ NMR spectrometer. We thank our colleagues Prof. Ged Parkin and Mr. Wesley Sattler for an X-ray structure analysis (see the Supplementary Information), and the National Science Foundation (CHE-0619638) is thanked for acquisition of an X-ray diffractometer.

\section{References}

1. Nakamura M, Hirai A, Sogi M, Nakamura E. Enantioselective addition of allylzinc reagent to alkynyl ketones. J. Am. Chem. Soc. 1998; 120:5846-5847.

2. Waltz KM, Gavenonis J, Walsh PJ. A simple, reliable, catalytic asymmetric allylation of ketones. Angew. Chem. Int. Ed. 2002; 41:3697-3699.

3. Wu TR, Shen L, Chong JM. Asymmetric allylboration of aldehydes and ketones using 3,3'disubstitutedbinaphthol-modified boronates. Org. Lett. 2004; 6:2701-2704. [PubMed: 15281748]

4. Canales E, Prasad KG, Soderquist JA. B-Allyl-10-Ph-9-borabicyclo[3.3.2]decanes: strategically designed for the asymmetric allylboration of ketones. J. Am. Chem. Soc. 2005; 127:11572-11573. [PubMed: 16104712]

5. Wadamoto M, Yamamoto H. Silver-catalyzed asymmetric Sakurai-Hosomi allylation of ketones. J. Am. Chem. Soc. 2005; 127:14556-14557. [PubMed: 16231892]

6. Miller JJ, Sigman MS. Design and synthesis of modular oxazoline ligands for the enantioselective chromium-catalyzed addition of allyl bromide to ketones. J. Am. Chem. Soc. 2007; 129:2752-2753. [PubMed: 17309263]

7. Barnett DS, Moquist PN, Schaus SE. The mechanism and an improved asymmetric allylboration of ketones catalyzed by chiral biphenols. Angew. Chem. Int. Ed. 2009; 48:8679-8682.

8. Shi S-L, Xu L-W, Oisaki K, Kanai M, Shibasaki M. Identification of modular chiral bisphosphines effective for $\mathrm{Cu}(\mathrm{I})$-catalyzed asymmetric allylation and propargylation of ketones. J. Am. Chem. Soc. 2010; 132:6638-6639. [PubMed: 20420398]

9. Robak MT, Herbage MA, Ellman JA. Synthesis and applications of tert-butanesulfinimide. Chem. Rev. 2010; 110:3600-3740. [PubMed: 20420386]

10. Pettit GR, Cichacz ZA, Gao F, Herald CL, Boyd MR, Schmidt JM, Hooper JNA. Isolation and structure of spongistatin 1. J. Org. Chem. 1993; 58:1302-1304.

11. Fusetani N, Shinoda K, Matsunaga S. Cinachyrolide A: a potent cytotoxic macrolide possessing two spiro ketals from marine sponge Cinachyra sp. J. Am. Chem. Soc. 1993; 115:3977-3981.

12. Kobayashi M, Aoki S, Sakai H, Kawazoe K, Kihara N, Sasaki T, Kitagawa I. Altohyrtin A, a potent anti-tumor macrolide from the Okinawan marine sponge Hyrtios altum. Tetrahedron Lett. 1993; 34:2795-2798.

13. Gaich T, Baran PS. Aiming for the ideal synthesis. J. Org. Chem. 2010; 75:4657-4673. [PubMed: 20540516]

14. Deng D-L, Lu Z-H. Monoallylation of symmetric diketones in the presence of water by allylic bromide and metallic zinc. Chinese Chem. Lett. 1994; 5:173-176.

15. Kira M, Sato K, Sekimoto K, Gewald R, Sakurai H. Stereoselective allylation of $\beta$-hydroxy- and $\beta$ amino-a, $\beta$-enones with allyltrifluorosilane/triethylamine systems. Chem. Lett. 1995:281-282.

16. Marton D, Stivanello D, Tagliavini G. Allylstannation of $\alpha-, \beta$ - and $\gamma$-diketones mediated by allylbutyltin halides: $\mathrm{Bu}_{2}\left(\mathrm{CH}_{2}=\mathrm{CHCH}_{2}\right) \mathrm{SnCl}$ and $\mathrm{Bu}\left(\mathrm{CH}_{2}=\mathrm{CHCH}_{2}\right) \mathrm{SnCl}_{2}$. J. Organomet. Chem. 1997; 540:77-81.

17. Leighton JL. Powerful and versatile silicon Lewis acids for Asymmetric chemical synthesis. Aldrichimica Acta. 2010; 43:3-12.

18. Kim H, Ho S, Leighton JL. A more comprehensive and highly practical solution to enantioselective aldehyde crotylation. J. Am. Chem. Soc. 2011; 133:6517-6520. [PubMed: 21486033] 
19. Burns NZ, Hackman BM, Ng PY, Powelson IA, Leighton JL. The enantioselective allylation and crotylation of sterically hindered and functionalized aryl ketones: convenient access to unusual tertiary carbinol structures. Angew. Chem. Int. Ed. 2006; 45:3811-3813.

20. Hackman BM, Lombardi PJ, Leighton JL. Highly diastereo- and enantioselective reagents for aldehyde crotylation. Org. Lett. 2004; 6:4375-4377. [PubMed: 15524487]

21. Spletstoser JT, Zacuto MJ, Leighton JL. Tandem silylformylation-crotylsilylation/Tamao oxidation of internal alkynes: a remarkable example of generating complexity from simplicity. Org. Lett. 2008; 10:5593-5596. [PubMed: 19007175]

22. Pinnavaia TJ, Collins WT, Howe JJ. Triorganosilicon acetylacetonates. Enol ether isomerism and stereochemical lability. J. Am. Chem. Soc. 1970; 92:4544-4550.

23. Reich HJ, Murcia DA. Stereochemical studies of degenerate silyl rearrangements. Stereospecificity of the tropolone and acetylacetone trialkylsilyl ether rearrangements. J. Am. Chem. Soc. 1973; 95:3418-3420.

24. Pinnavaia TJ, McClarin JA. Rearrangements of 1-acetylacetonato-1-methyl-1-silacyclobutane via internal nucleophilic displacement. J. Am. Chem. Soc. 1974; 96:3012-3013.

25. McClarin JA, Schwartz A, Pinnavaia TJ. 1,5-Migrations of silicon between oxygen centers in silyl $\beta$-diketones. J. Organomet. Chem. 1980; 188:129-139.

26. Seeman JI. Effect of conformational change on reactivity in organic chemistry. Evaluations, applications, and extensions of Curtin-Hammett Winstein-Holness kinetics. Chem. Rev. 1983; 83:83-134.

27. Mirarchi D, Ritchie GLD. Solution-state conformations of 2-fluoro-, 2-chloro- and 2-bromoacetophenone: a diploe moment and Kerr effect study. J. Mol. Struct. 1984; 118:303-310.

28. Kulhánek J, Böhm S, Palát K Jr. Exner O. Steric inhibition of resonance: revision of the principle on the electronic spectra of methyl-substituted acetophenones. J. Phys. Org. Chem. 2004; 17:686693.

29. Cheng CL, Ritchie GLD. Conformations of the formyl-, acetyl-, and benzoyl-pyridines. J. Chem. Soc., Perkin Trans. 1973; 2:1461-1465.

30. Ramachandran PV, Gong B, Brown HC, Francisco JS. Relationship between the structure and enantioselectivity in the asymmetric reduction of $2^{\prime}, 6^{\prime}$-disubstituted acetophenones with DIPchloride $^{\mathrm{TM}}$. An ab initio study. Tetrahedron Lett. 2004; 45:2603-2605. 
a<smiles>C=CCC(C)(O)CC(C)=O</smiles><smiles>C=CCC(O)(CC(C)=O)CC(C)=O</smiles>

Direct asymmetric allylation of acetylacetone?

C

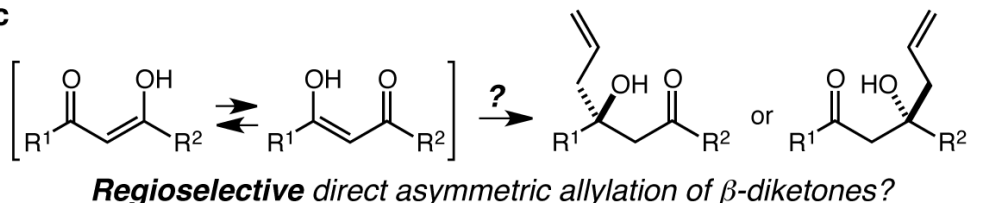

Figure 1. Origin and evolution of a proposal for a direct, enantioselective, and regioselective allylation of $\beta$-diketones

a Retrosynthesis of the $\mathrm{AB}$ spiroketal of spongistatin 1 to $\beta$-hydroxyketone 1 . b A direct asymmetric allylation of acetylacetone would have to contend with the enol form of the diketone. $\mathbf{c}$ The generalization of a direct method would require control of regioselectivity. 
a

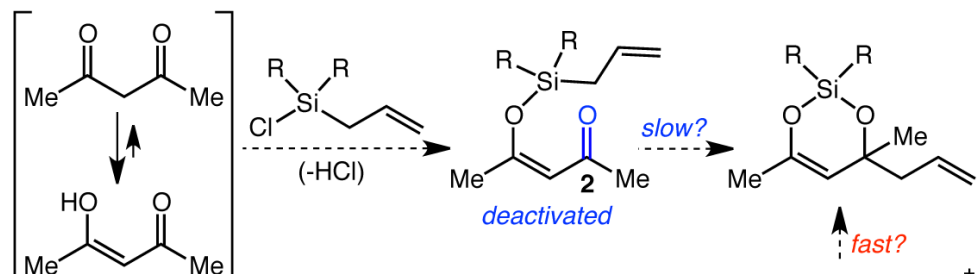

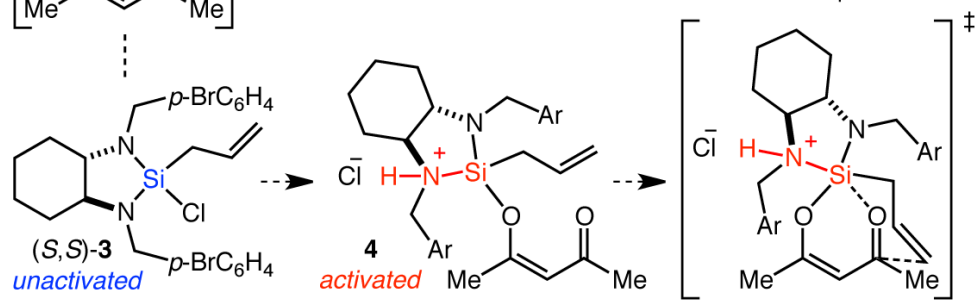

b

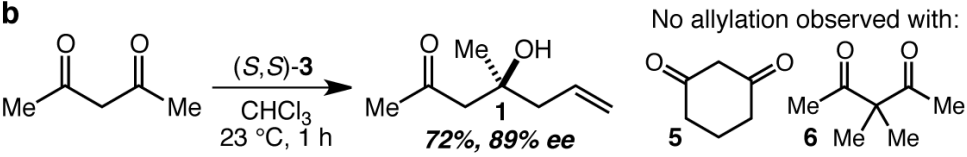

Figure 2. Reaction design and proof of concept

a The reaction design entails tethering the allylsilane to the enol form of the diketone and activation of the silane by the liberated $\mathrm{HCl}$. b First demonstration of the asymmetric allylation of acetylacetone. 

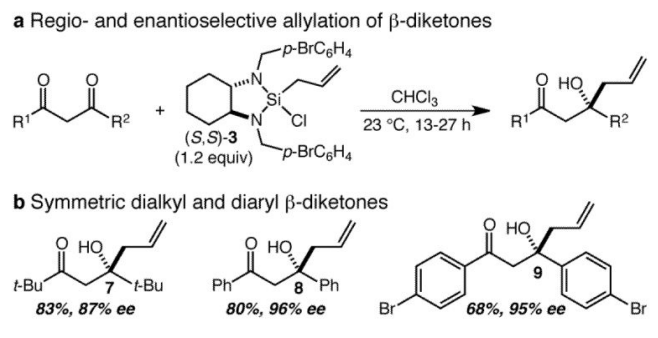

c Unsymmetrical aryl, alkyl $\beta$-diketones
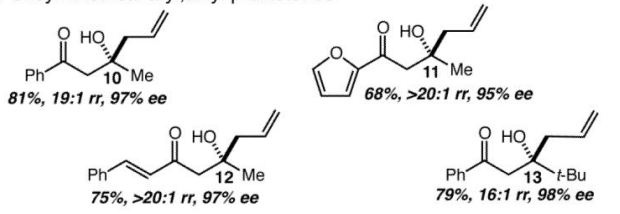

d Unsymmetrical diaryl $\beta$-diketones
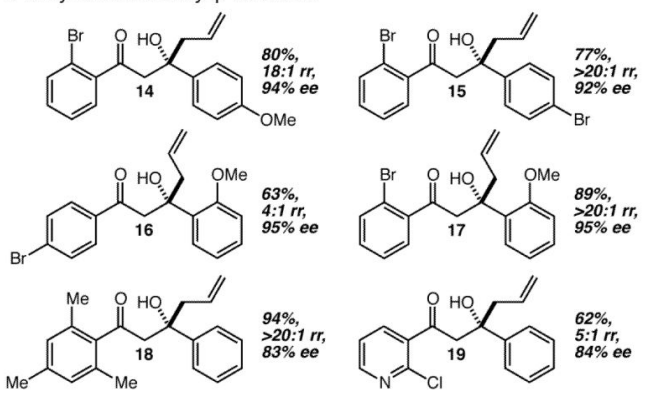

Figure 3. Scope of the regio- and enantioselective allylation of $\beta$-diketones

a The allylation reactions were conducted by treating the $\beta$-diketone with 1.2 equiv of $(S, S)-3$ in $\mathrm{CHCl}_{3}$, and stirring the resultant mixture at ambient temperature for 13-27 h. In all cases the reported yield refers to the yield of isolated ( $\geq 20: 1$ regioisomeric purity) major product after work-up and purification by flash chromatography, and the enantiomeric excess (ee) was determined by chiral high-performance liquid chromatography. $\mathbf{b}$ Enantioselective allylation of symmetrical $\beta$-diketones. $\mathbf{c}$ Regio- and enantioselective allylation of unsymmetrical aryl,alkyl $\beta$-diketones ( $\mathrm{rr}=$ regioisomer ratio). $\mathbf{d}$ Regio- and enantioselective allylation of unsymmetrical diaryl $\beta$-diketones. 
a

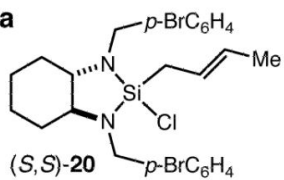

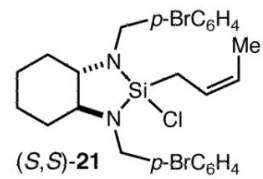<smiles>Cc1cc(-c2ccccc2)cc(O)c1C(=O)c1ccccc1</smiles><smiles>[R]C(=O)CC(=O)NC(C)(C)C</smiles><smiles>C1CC[Te]CC1</smiles><smiles>C=CC(C)C(O)(CC(C)=O)C(C)(O)CC(C)=O</smiles>

b

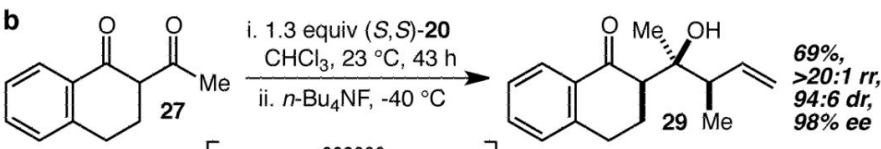
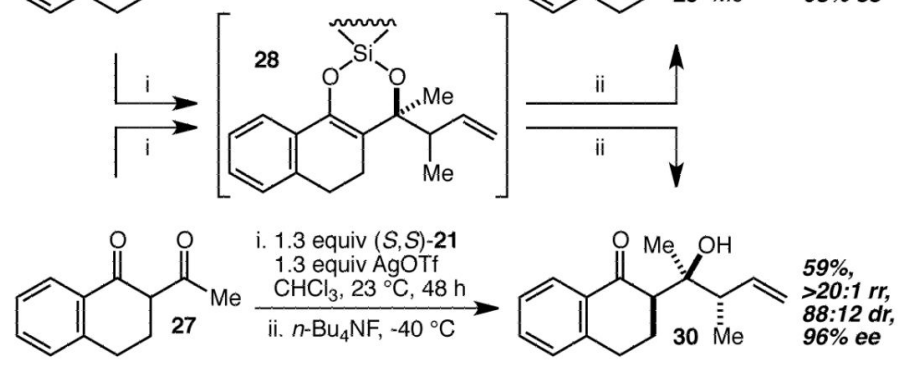

Figure 4. Regio- diastereo- and enantioselective crotylation of $\beta$-diketones a The crotylation of $\beta$-diketones allows the establishment of a second stereocenter in the homoallylic alcohol products. b a-Substitution on the $\beta$-diketone substrate may be parlayed into reactions that establish three stereocenters from simple starting materials. 

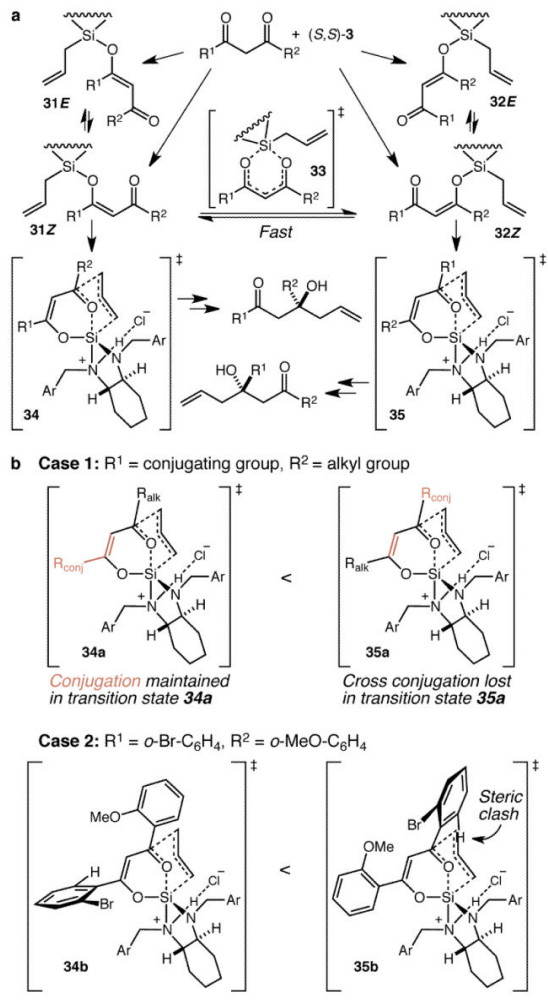

Figure 5. General mechanism and origin of regioselectivity

a A general mechanistic scheme for the allylation of $\beta$-diketones wherein the product distribution is determined by Curtin-Hammett kinetics. b Models for the observed regioselectivitiy. 